\title{
Discarded candidate companions to low-mass members of Chamaeleon I ${ }^{\star}$ (Research Note)
}

\author{
F. Comerón ${ }^{\star \star}$ \\ ESO, Karl-Schwarzschild-Strasse 2, 85748 Garching bei München, Germany \\ e-mail: fcomeron@eso.org
}

Received 28 June 2011 / Accepted 8 November 2011

\begin{abstract}
Context. Direct detections of brown dwarfs and planetary-mass companions to members of nearby star-forming regions provide important clues about the process of star formation, core fragmentation, and protoplanetary disk evolution.

Aims. We study two faint objects at a very small angular distance from the low-mass star ESO-H $\alpha-558$ and the possible massive brown dwarf ESO-H $\alpha-566$, both of which are members of the Chamaeleon I star-forming region, to establish whether they are physical companions to those sources. If they are, their low luminosities should imply L or T spectral types, which have clearly detectable spectral features.

Methods. Adaptive optics-assisted imaging and spectroscopy of both faint candidate companions has been obtained with the NACO instrument at the Very Large Telescope (VLT).

Results. Photometry shows that the colors of both objects are compatible with them being moderately reddened, normal stars in the background of the Chamaeleon I clouds. This interpretation is confirmed spectroscopically, as the spectrum between 1.4 and $2.4 \mu \mathrm{m}$ of both objects has a featureless, monotonic slope lacking the strong $\mathrm{H}_{2} \mathrm{O}$ absorption features that dominate cool stellar and substellar spectra in that domain.

Conclusions. We demonstrate that the two faint sources seen very close to ESO-H $\alpha-558$ and ESO-H $\alpha-566$ are unrelated background stars, instead of giant planetary-mass companions as might be expected based on their faintness and angular proximity.
\end{abstract}

Key words. brown dwarfs - stars: formation - stars: pre-main sequence - binaries: general - stars: low-mass

\section{Introduction}

Observations in recent years have demonstrated that the direct detection of low-mass brown dwarf and giant planet-mass companions to stars is achievable (e.g. Neuhäuser et al. 2004; Chauvin et al. 2005; Kasper et al. 2007; Lafrenière et al. 2008a; Kalas et al. 2008; Fukagawa et al. 2009; Lagrange et al. 2010; Stumpf et al. 2010). Statistically significant samples of these objects are fundamental to understand their origin, justifying the large amounts of time invested using large facilities for their discovery and characterization. Searches for companions in the vicinity of members of star-forming regions (Itoh et al. 2005; Todorov et al. 2010; Biller et al. 2011; Ireland et al. 2011; Kuzuhara et al. 2011) are of special interest, owing to the insight that they may provide into the timescale for planet formation and early dynamical evolution of planetary systems. The confirmation of the physical companionship of faint objects ideally requires observations collected over a time span long enough to establish common motion across space and perhaps even orbital motion (Neuhäuser et al. 2010). Alternatively, strong evidence of candidate very low-mass companions can be derived from their spectroscopic characteristics, if they are found to be consistent

* Based on observations collected with the Very Large Telescope (VLT) at the European Southern Observatory, Paranal, Chile, under observing programmes 075.C-0809(B) and 078.C-0429(C).

$\star \star$ Visiting astronomer at the Vatican Observatory. with the expectations derived from the luminosity, age, and distance inferred from the primary component of the system.

The Chamaeleon I star-forming region (Luhman 2008) has become a target of searches for low-mass companions. Its stellar population has been established down to the substellar regime to a very high degree of completeness (Luhman 2007; Mužic et al. 2011). At its distance of 160 pc (Luhman 2007), adaptive optics allows the direct imaging of companions only a few times as massive as Jupiter at distances of a few tens of AU from the central object. Low-mass binary companions to Chamaeleon I members have been reported by Kraus \& Hillenbrand (2007), Lafrenière et al. (2008b), and Huélamo et al. (2011).

This Note reports on apparently promising candidate companions of two of the Chamaeleon I members discovered by Comerón et al. (2004), ESO-H $\alpha-558$ and ESO-H $\alpha-566$. The close angular proximity of the candidate companions to their possible central objects, and their apparent brightness, indicative of masses a few times that of Jupiter at most if they are true companions, make them very attractive targets for followup observations to confirm their physical relationship to the Chamaeleon I members. However, the adaptive optics-assisted imaging and spectroscopy presented here definitely exclude both relationships by demonstrating instead that the two objects are background stars that just happen to be closely aligned with the lines of sight to ESO-H $\alpha-558$ and ESO-H $\alpha$ 566. These negative results should discourage other researchers planning deep 
Table 1. Companion detection limits $(5 \sigma)$ in $K_{\mathrm{S}}$ in the ISAAC observations.

\begin{tabular}{lcccccc}
\hline \hline ESO-H $\alpha$ & Primary & \multicolumn{5}{c}{ Distance (arcsec) } \\
& (2MASS) & 0.75 & 1.0 & 1.0 & 2.0 & 3.0 \\
\hline 552 & 10.801 & 16.9 & 17.2 & 17.5 & 18.0 & 18.2 \\
554 & 13.545 & 16.8 & 17.6 & 18.4 & 19.0 & 18.9 \\
557 & 10.903 & 15.9 & 18.0 & 18.4 & 18.3 & 18.1 \\
558 & 10.642 & 17.2 & 17.7 & 17.8 & 18.0 & 17.9 \\
559 & 11.486 & 13.2 & 14.4 & 16.4 & 17.8 & 19.2 \\
560 & 11.027 & 16.7 & 17.4 & 17.9 & 18.6 & 18.9 \\
561 & 11.167 & 15.4 & 16.0 & 17.7 & 18.4 & 18.7 \\
564 & 11.439 & 14.9 & 15.9 & 17.2 & 17.8 & 18.0 \\
565 & 10.552 & 14.8 & 15.8 & 16.9 & 17.4 & 17.8 \\
566 & 11.033 & 17.0 & 17.2 & 17.9 & 18.4 & 18.9 \\
567 & 10.236 & 15.0 & 16.0 & 17.4 & 17.8 & 17.8 \\
568 & 10.748 & 14.7 & 15.5 & 17.9 & 17.8 & 17.7 \\
569 & 14.579 & 15.5 & 16.3 & 17.5 & 18.1 & 18.8 \\
570 & 11.842 & 16.9 & 17.9 & 18.4 & 18.6 & 18.9 \\
571 & 11.607 & 14.2 & 15.3 & 17.6 & 18.5 & 18.5 \\
572 & 10.824 & 17.1 & 18.2 & 19.0 & 18.9 & 18.9 \\
574 & 14.606 & 16.7 & 17.7 & 18.7 & 19.0 & 19.2 \\
\hline
\end{tabular}

searches for companions of Chamaeleon I from investing precious telescope time in the duplication of the observations presented here.

\section{Observations}

A set of $K_{\mathrm{S}}$-band images of 17 Chamaeleon I members reported by Comerón et al. (2004) was obtained in service mode using the ISAAC infrared imager and spectrograph (Moorwood 1997) at the ESO Very Large Telescope (VLT) between 27 March and 28 April 2005. The observations of each target consisted of exposures totaling $3.7 \mathrm{~min}$ of integration time, and the field imaged around each target covered $4.9 \mathrm{arcmin}^{2}$. The limits for the detection of companions in the processed ISAAC images were estimated by adding artificial stars within a range of magnitudes at selected distances from the central object, after subtraction at the position of the latter of a point-spread function (PSF) constructed from the images of other bright isolated stars in the same field of view. The same PSF was used for the simulated companion, after scaling to its selected magnitude. PSF photometry of the simulated companion was then obtained, and the difference between the input and recovered magnitudes was computed. By repeating the procedure for a variety of position angles of the simulated companion with respect to the central object, the $5 \sigma$ detection limit at each distance of the PSF-subtracted central object was thus derived. Results are given in Table 1 . The variations in limiting magnitude and its dependence with the distance to the central object are a consequence of the differences in the conditions, mostly atmospheric seeing, under which the different targets were observed.

Two of the targets observed, ESO-H $\alpha 558$ and ESO-H $\alpha$ 566, turned out to have faint companions at distances that appear at first sight to be significantly closer than the average distance between neighboring stars in their respective fields. The properties of ESO-H $\alpha-558$ and ESO-H $\alpha-566$ inferred by means of premain sequence evolutionary track fitting were given in Comerón et al. (2004). The star ESO-H $\alpha-558$ is of a low-mass and a spectral type M4.5 with an estimated mass of $0.2 M_{\odot}$, whereas ESO$\mathrm{H} \alpha-566$ has a spectral type M6.5 that places it close to the substellar boundary with a mass around $0.08 M_{\odot}$. Both objects are similarly obscured by foreground dust. Despite their differences in mass and temperature, $\mathrm{ESO}-\mathrm{H} \alpha 558$ is only 0.4 mag brighter than ESO-H $\alpha 566$ in $K_{\mathrm{S}}$, probably indicating that ESO-H $\alpha-558$ is older. On the basis of the models of Baraffe et al. (1998), Comerón et al. (2004) assigned an age of 3 Myr to ESO-H $\alpha$ 558 and 1 Myr to ESO-H $\alpha-566$. Kraus \& Hillenbrand (2007) reported that $\mathrm{ESO}-\mathrm{H} \alpha-566$ has a wide binary companion at a distance of 23.65" away.

ESO-H $\alpha-558$ and ESO-H $\alpha-566$ were thus selected for follow-up with NACO, the adaptive optics infrared imager and spectrograph at the VLT (Lenzen et al. 2003; Rousset et al. 2003). Both targets are bright enough to be used for wavefront sensing in the infrared. Dichroic mirrors allowing the transmission of $80 \%$ of the infrared light to the camera were used, with the remaining $20 \%$ being used for wavefront sensing. Images were obtained using the camera optics that provided the smallest available pixel scale, resulting in a field of view of $13^{\prime \prime}$. The star ESO-H $\alpha-558$ was observed on 23 and 24 February 2007, and ESO-H $\alpha-566$ on 4 March 2007. Equivalent exposures of 525,441 , and $448 \mathrm{~s}$ were obtained through the $J, H$ and $K_{\mathrm{S}}$ filters, respectively. Our PSF instrumental photometry was then performed on both the primary target and the candidate companion using the primary ${ }^{1}$ to determine the shape of the PSF. Owing to the close proximity of both objects, no significant variation in the PSF between their respective positions was expected. The photometric zeropoint was then derived by taking the cataloged 2MASS magnitudes (Skrutskie et al. 2006) of each star (both being far from saturating the detector) as a reference to determine the magnitudes of the candidate companions. The NACO images were also used to accurately determine the distance and position angle of the companions, which are listed in Table 2.

The similar degree of crowdedness of the fields around our 17 ISAAC targets allows us to estimate the likelihood of the candidate companions being actually the result of a chance alignment of their primaries with background stars. A representative average surface density of 0.0031 objects per $\operatorname{arcsec}^{2}$ with $12<K_{\mathrm{S}}<18$ is found. The brighter limit corresponds to the approximate magnitude of a massive brown dwarf with an age of $3 \mathrm{Myr}$, whereas the fainter limit is a typical $5 \sigma$ detection limit at $2^{\prime \prime}$ from the central object in the observed fields, corresponding to a $3 M_{\text {Jup }}$ object of that same age. This is thus the approximate magnitude range of potential substellar companions detectable in our observations. Following an approach similar to that of Béjar et al. (2008), the probability $P$ of finding more than a certain number $n$ of background stars within a distance $d$ to any of the 17 Chamaeleon I members observed is given by Poisson statistics:

$P(>n)=1-\sum_{0}^{n} \frac{\lambda^{n}}{n !} \mathrm{e}^{-\lambda}$

where

$\lambda=17 \times 0.0031 \operatorname{arcsec}^{-2} \pi d^{2}$.

The probability of finding the first background star within 1."68 of any primary (the distance of the candidate companion to ESO$\mathrm{H} \alpha-566)$ is thus found to be 0.38 , whereas the probability of finding a second star within 2 '. 06 of the same or another primary drops to 0.14 . Although not a compelling evidence, this

1 The term "primary" is used throughout this paper for convenience to refer to $\mathrm{ESO}-\mathrm{H} \alpha-558$ and $\mathrm{ESO}-\mathrm{H} \alpha-566$. It is not meant to imply that they are the actually predominant member of a physical system, which would be the case only if the faint sources close to them were confirmed to be true companions. 
Table 2. Photometry and location of the candidate companions.

\begin{tabular}{lccccc}
\hline \hline ESO-H $\alpha$ & $J$ & $H$ & $K_{\mathrm{S}}$ & $\rho\left({ }^{\prime \prime}\right)$ & $\theta\left({ }^{\circ}\right)$ \\
\hline 558 main & $11.81 \pm 0.02$ & $11.00 \pm 0.02$ & $10.64 \pm 0.02$ & - & - \\
558 comp. & $19.51 \pm 0.2$ & $18.11 \pm 0.1$ & $17.55 \pm 0.1$ & $2.06 \pm 0.03$ & $135.4 \pm 0.6$ \\
& & & & - \\
566 main & $12.36 \pm 0.02$ & $11.45 \pm 0.02$ & $11.033 \pm 0.02$ & - & - \\
566 comp. & $18.82 \pm 0.15$ & $17.67 \pm 0.1$ & $17.13 \pm 0.1$ & $1.68 \pm 0.02$ & $193.8 \pm 0.8$ \\
\hline
\end{tabular}
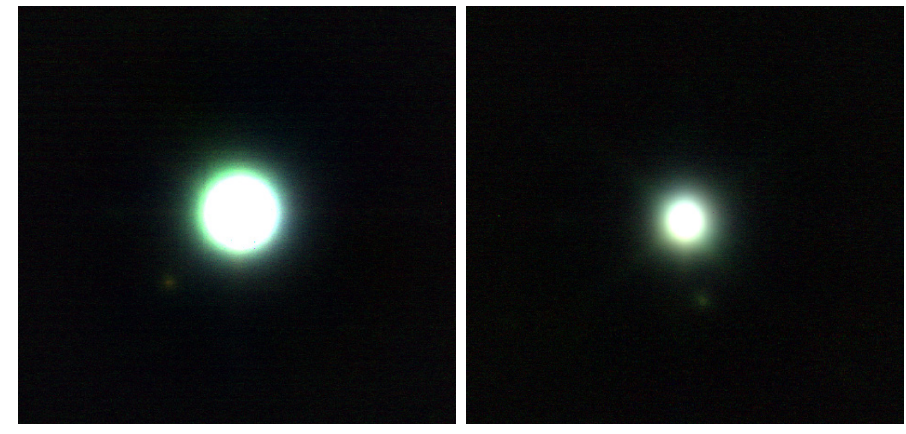

Fig. 1. $J H K_{\mathrm{S}}$ composite (in color in the electronic version) of ESO-H $\alpha-558$ (left), ESO-H $\alpha-566$ (right) and their candidate companions. The separations between the primaries and their candidate companions are measured to be 2.'06 (ESO-H $\alpha-558)$ and 1'.68 (ESO-H $\alpha-566)$. North is up and east to the left.

does suggest that our finding of two sources at such short distances from their primaries may be due to a physical relationship with them, rather than to a chance alignment. For this reason, NACO spectroscopy of both sources was obtained on the nights of 23 February 2007 (ESO-H $\alpha$-558) and 2 April 2007 (ESO-H $\alpha$-566). The spectra have a resolution of $\lambda / \Delta \lambda \simeq 450$ and provide simultaneous coverage of the $H$ and $K$ bands. The slit was oriented so as to include both the primary and the candidate companion. Equivalent exposure times were $45 \mathrm{~min}$ for ESO-H $\alpha-558$, and 60 min for ESO-H $\alpha-566$. The observing conditions permitted a higher quality adaptive optics correction to be made for data acquired on the second night, resulting in a significantly higher quality spectrum of the candidate companion of ESO-H $\alpha$-566. The signal-to-noise ratios at $1.5 \mu \mathrm{m}$ (near the peak emission) per resolution element are 3 for ESO-H $\alpha-558$ and 10 for $\mathrm{ESO}-\mathrm{H} \alpha-566$. A Gaussian function was fitted to the spectral trace of the primary at each wavelength and then subtracted, in order to remove the PSF wings of the spectrum of the primary at the spatial position of that of the candidate companion. The extracted spectra were corrected for telluric absorption by dividing them by the spectrum of the B9.5IV star Hip 57374, which is located few degrees away from the targets, and was observed close in time and at essentially the same airmass.

\section{Results and conclusions}

NACO images of ESO-H $\alpha-558$ and ESO-H $\alpha-566$ and their candidate companions are presented in Fig. 1, and their photometry and that of their primaries (the latter taken from 2MASS) are given in Table 2 . The distance $\rho$ and position angle $\theta$, measured from north to east, of the candidate companions to their primaries are also given there. The NACO observations show no other objects of similar brightness within a radius of at least $10^{\prime \prime}$ from each source.

Adopting a distance modulus $D M=6.05$ (Luhman 2007) and $J$-band extinctions $A_{J}=0.24$ and $A_{J}=0.49$ derived respectively for $\mathrm{ESO}-\mathrm{H} \alpha-558$ and $\mathrm{ESO}-\mathrm{H} \alpha-566$ by Comerón et al. (2004), the absolute $K_{\mathrm{S}}$ magnitudes of the candidate companions to ESO-H $\alpha$-558 and ESO-H $\alpha$-566 would be $M_{K} \simeq 11.4$ and $M_{K} \simeq 10.8$, respectively, if they were true members of the star formation region. The respective projected distances to their primaries would be 273 AU and 334 AU. Their colors are only moderately red, which is inconsistent with dustrich atmospheres (Allard et al. 2001). However, this might be explained if the objects were cool enough for their atmospheres to be dust-depleted owing to the sedimentation of dust grains below the photosphere (Burrows 2009), which has been proposed to mark the transition between the $\mathrm{L}$ and $\mathrm{T}$ spectral types (Kirkpatrick 2005). The effective temperature at which the transition is expected to happen, $T_{\text {eff }} \simeq 1400 \mathrm{~K}$, is reached by objects of a few millions of years of age at luminosities consistent with those derived above for the two candidate companions (Baraffe et al. 2003). The colors are somewhat redder than predicted for these objects, particularly $J-H$, even accounting for the foreground extinction estimated from the colors of the primary. However, large uncertainties in the predicted broad-band colors of ultracool objects still subsist (e.g. Zapatero Osorio et al. 2008) and this difference should not be taken as a definitive argument against the companion nature of both objects. On the other hand, the colors are also consistent with those of normal background stars reddened by the intervening cloud, and the photometry of 2MASS sources within $2^{\prime}$ of each target indicate that the $K$-band extinction levels caused by the cloud on the background do not exceed $A_{K} \sim 0.3-0.4$, resulting in color excesses $E\left[H-K_{\mathrm{S}}\right] \sim 0.2$ and $E[J-H] \sim 0.5$.

Spectroscopy can provide definitive confirmation of the true nature of the companion candidates, given the existence of strong water vapor absorption bands with broad wings that dominate the shape of the near-infrared spectral energy distribution at effective temperatures near and below $\sim 3500 \mathrm{~K}$. Those bands are clearly visible in the spectra of the primaries (Fig. 2), which confirm the M4.5 and M6.5 types assigned by Comerón et al. (2004) based on spectroscopy in the visible. If the candidate companions were T-type as hinted by the photometry, one would also expect $\mathrm{CH}_{4}$ bands to be present in the spectrum. Despite the faintness of the companions, the prominence of those features should make them clearly visible in the $H$ and $K$ spectra, and be even more pronounced than in the spectra of the primaries.

Figure 3 shows that this is not the case for any of the candidate companions. Despite the low signal-to-noise ratio, especially for the candidate companion of ESO-H $\alpha-558$, the overall shape of the spectral energy distribution of both objects is found to be essentially featureless. A detailed classification is impossible, but the clear lack of water vapor absorption indicates spectral types earlier than $\mathrm{M}$, thus ruling out these objects as true companions and members of Chamaeleon I.

On visual inspection, the objects in the close vicinity of ESO-H $\alpha-558$ and ESO-H $\alpha-566$ are tantalizing sources. The crowding level of both fields is rather low, thanks to both their Galactic latitude and to the natural screen on the background 


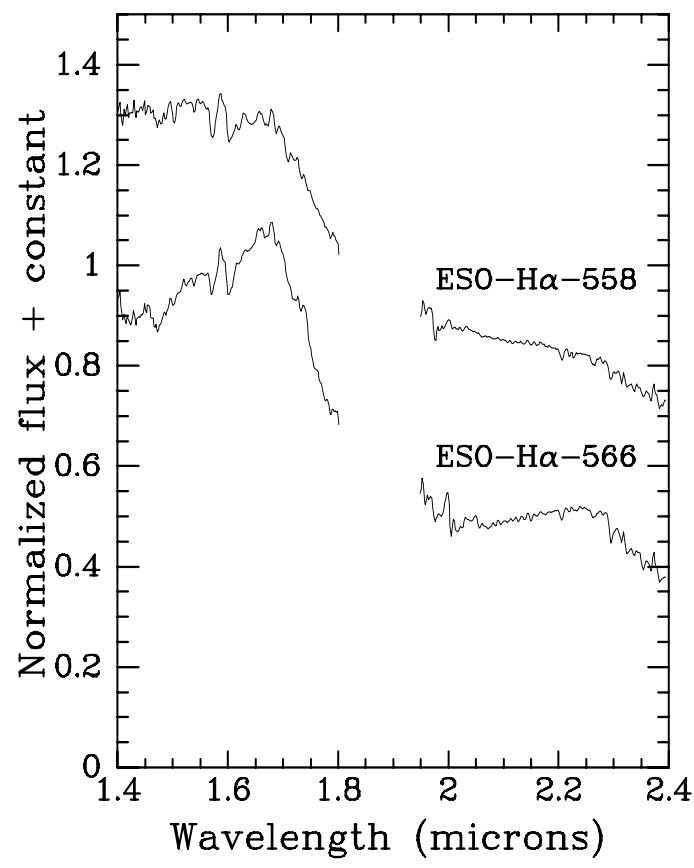

Fig. 2. Near-infrared spectra of ESO-H $\alpha-558$ and ESO-H $\alpha-566$ obtained with NACO, clearly showing the differences in the shapes of their spectral energy due to the later spectral type of ESO-H $\alpha-566$. The "triangular" shape of the spectrum in the $H$ band, with a distinct change of slope near $1.7 \mu \mathrm{m}$, is characteristic of cool young objects with low surface gravity.

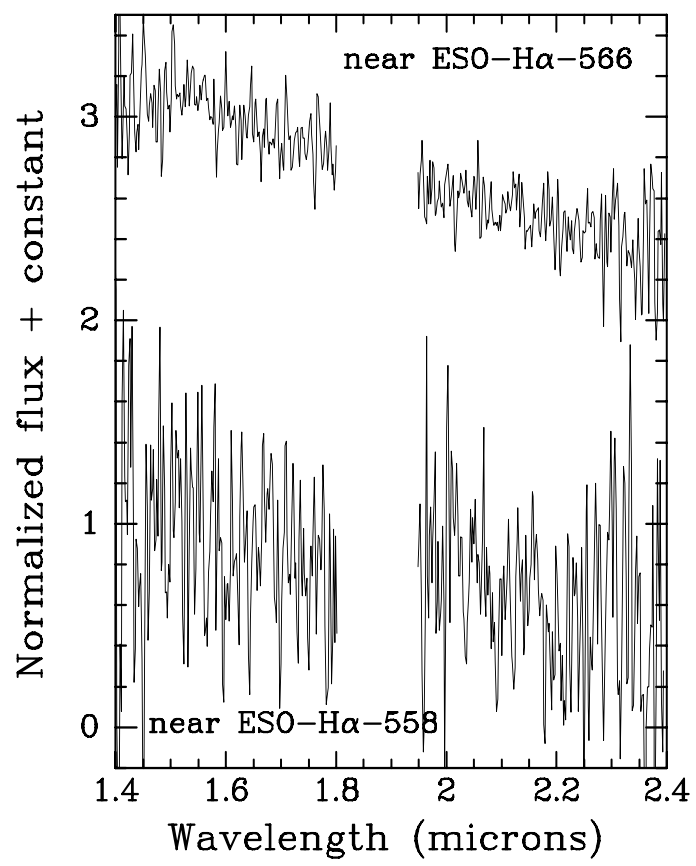

Fig. 3. Near-infrared spectra of the two candidate companions. Despite the low signal-to-noise ratio, it is clear that none of the objects displays the strong absorption features due to $\mathrm{H}_{2} \mathrm{O}$ and possibly $\mathrm{CH}_{4}$ expected from photospheres colder than those of the primary sources. The featureless slope with no hints of $\mathrm{H}_{2} \mathrm{O}$ absorption bands indicates temperatures above $3500 \mathrm{~K}$, demonstrating that both objects are normal stars located beyond the Chamaeleon I clouds and casually aligned along the line of sight to ESO-H $\alpha-558$ and ESO-H $\alpha-566$. provided by dust extinction in their direction, thus reinforcing the impression against a casual arrangement of unrelated sources along the line of sight. In this respect, the finding that both objects can be confidently discarded as true companions is somewhat disappointing. However, it does illustrate the vital importance of follow-up observations, and it is to be hoped that the results presented here will ensure that future telescope time at large facilities currently needed to carry out similar observations is indeed invested for other promising sources, rather than these two.

Acknowledgements. This publication makes use of data products from the Two Micron All Sky Survey, which is a joint project of the University of Massachusetts and the Infrared Processing and Analysis Center/California Institute of Technology, funded by the National Aeronautics and Space Administration and the National Science Foundation. I am indebted to Bo Reipurth for a critical reading of an earlier version of this manuscript and for several suggestions leading to improvements of this Research Note. I also thank the anonymous referee for comments leading to further substantial improvements. I am pleased to thank the Paranal Science Operations staff for the execution of the observations in Service Mode, and Lowell Tacconi-Garman at the ESO's User Support Department, within the ESO's Data Management and Operations Division, for the support with the Phase 2 preparation of the NACO observations. Much of this work was carried out during a science visit at the Specola Vaticana, whose hospitality is gratefully acknowledged.

\section{References}

Allard, F., Hauschildt, P. H., Alexander, D. R., Tamanai, A., \& Schweitzer, A. 2001, ApJ, 556, 357

Baraffe, I., Chabrier, G., Allard, F., \& Hauschildt, P. H. 1998, A\&A, 337, 403

Baraffe, I., Chabrier, G., Barma, T. S., Allard, F., \& Hauschildt, P. H. 2003, A\&A, 402, 701

Béjar, V. J. S., Zapatero Osorio, M. R., Pérez-Garrido, A., et al. 2008, ApJ, 673, L185

Biller, B., Allers, K., Liu, M., Close, L. M., \& Dupuy, T. 2011, ApJ, 730, 39

Burrows, A. 2009, in Cosmic Dust -Near and Far, ASP Conf. Ser., 414

Chauvin, G., Lagrange, A.-M., Dumas, C., et al. 2005, A\&A, 38, L25

Comerón, F., Reipurth, B., Henry, A., \& Fernández, M. 2004, A\&A, 417, 583

Fukagawa, M., Itoh, Y., Tamura, M., et al. 2009, ApJ, 696, L1

Huélamo, N., Lacour, S., Tuthill, P., et al. 2011, A\&A, 528, L7

Ireland, M. J., Kraus, A., Martinache, F. L., \& Hillenbrand, L. A. 2011, ApJ, 726,113

Itoh, Y., Hayashi, M., Tamura, M., et al. 2005, ApJ, 620, 984

Kalas, P., Graham, J. R., Chiang, E., et al. 2008, Science, 322, 1345

Kasper, M., Apai, D., Janson, M., \& Brandner, W. 2007, A\&A, 472, 321

Kirkpatrick, J. D. 2005, ARA\&A, 43, 195

Kraus, A. L., \& Hillenbrand, L. A. 2007, ApJ, 662, 413

Kuzuhara, M., Tamura, M., Ishii, M., et al. 2011, AJ, 141, 119

Lafrenière, D., Jayawardhana, R., \& van Kerkwijk, M. H. 2008a, ApJ, 689, L153

Lafrenière, D., Jayawardhana, R., Brandeker, A., Ahmic, M., \& van Kerkwijk, M. H. 2008b, ApJ, 683, 844

Lagrange, A.-M., Bonnefoy, M., Chauvin, G., et al. 2010, Science, 329, 57

Lenzen, R., Hartung, M., Brandner, W., et al. 2003, SPIE, 4841, 944

Luhman, K. L. 2007, ApJS, 173, 104

Luhman, K. L. 2008, Chamaeleon, in Handbook of Star Forming Regions, ed.

B. Reipurth, ASP Monographs, 2

Moorwood, A. F. 1997, SPIE, 2871, 1146

Mužić, K., Scholz, A., Geers, V., Fissel, L., \& Jayawardhana, R. 2011, ApJ, 732, 86

Neuhäuser, R., \& Guenther, E. W. 2004, A\&A, 420, 647

Neuhäuser, R., Schmidt, T. O. B., Hambaryan, V. V., \& Vogt, N. 2010, A\&A, 516, A112

Rousset, G., Lacombe, F., Puget, P., et al. 2003, SPIE, 4839, 140

Skrutskie, M. F., Cutri, R. M., Stiening, R., et al. 2006, AJ, 131, 1163

Stumpf, M. B., Brandner, W., Joergens, V., et al. 2010, ApJ, 724, 1

Todorov, K., Luhman, K. L., \& McLeod, K. K. 2010, ApJ, 714, L84

Zapatero Osorio, M. R., Béjar, V. J. S., Bihain, G., et al. 2008, A\&A, 477, 895 